\title{
sciendo
}

RESEARCH PAPERS FACULTY OF MATERIALS

SCIENCE AND TECHNOLOGY IN TRNAVA

SLOVAK UNIVERSITY OF TECHNOLOGY

IN BRATISLAVA

2019, Volume 27, Number 45

DOI 10.2478/rput-2019-0026

\section{PRODUCT CLASSIFICATION USING NEURAL NETWORK AT INDUSTRY ROBOTIC LINE}

\author{
Igor HALENÁR ${ }^{1}$, Gabriela KRIŽANOVÁ ${ }^{1}$ \\ ${ }^{1}$ SLOVAK UNIVERSITY OF TECHNOLOGY IN BRATISLAVA \\ FACULTY OF MATERIALS SCIENCE AND TECHNOLOGY IN TRNAVA \\ INSTITUTE OF APPLIED INFORMATICS, AUTOMATION AND MECHATRONICS \\ UliCA JÁNA BOTTU Č. 2781/25, 91724 TRNAVA, SLOVAK REPUBLIC \\ e-mail: igor.halenar@stuba.sk, gabriela.krizanova@stuba.sk \\ Received 27 August 2019, accepted 24 September 2019, published 29 November 2019
}

\begin{abstract}
The article describes a possible way of implementing a neural network in recognizing the shape and position of the products in the production process. The neural network is designed as a multilayer perceptron (MLP), and the whole system is implemented in a form of attachment to robotic arm, where the primary task of neural network is to distinguish a position of product. The neural network is trained like a classifier and outputs are used to control the robot. The advantage of the solution is a high degree of reliability of product positioning under different lighting conditions.
\end{abstract}

\section{Key words}

Neural network, Deep learning, Detection, Robot

\section{INTRODUCTION}

Nowadays, the industry manufacturing copes with rapid changes. Implementation of the Industry 4.0 principles in technical practice induces the introduction of new management methods. Those new methods of data processing include progressive ways such as machine learning, pattern recognition, deep learning, data mining, IOT communication, cloud computing and process virtualization. The current trend, thanks to these new approaches, is to increase the volume of data owing to the more often image and graphical data processing in production management. Processing such type of data is difficult and it means using effective methods of image processing. It represents mainly the use of neural networks for analysis and pattern recognition. Outputs from neural networks are used to control real processes in real environment. Automated robotic production lines make it possible to find a lot of examples of neural networks utilization for image recognition. Similar problem is also dealt with in our research. The main objective is to solve image processing in real conditions of a robotized line. 


\section{THEORETICAL FOUNDAMENTALS AND RELATED RESEARCH}

At present, the character of the image recognition technology has become a hot research topic in the field of artificial intelligence and pattern recognition [5]. Thanks to new technologies, the field of pattern recognition keeps evolving, improving and often is used in solving practical problems. Many systems used today in science and everyday life cannot exist without a form of implementation of pattern recognition algorithms or embedded decision rules. Pattern recognition has especially found application in the field of robotics. Recent researches in robotics develop in the direction of increasing robot autonomy and independence in working tasks. Owing to their characteristics of learning, memory and generalization, neural networks are chosen as a suitable method for solving problems of recognition and classification of geometric shapes, image, etc. [1]. The recognition problem in a real industrial setting is significantly more challenging than character recognition in documents or natural scenes [7].

The areas of recognition and classification of geometric shapes may be of interest for implementation of many robotic tasks, especially those related to gripping objects by a robotic arm or movement of a robot across a set of obstacles [1].

There are various methods devoted to this issue. A group of authors used novel method which was based on the transfer learning technique proposed to classify the spot-welding products according to their solder joint images. The GoogLeNet was used to extract the features of the solder joint image, which is pretrained on the ImageNet. Then a multilayer perceptron (MLP) was used to classify those images [2].

The other research presented in this area refers to classification of geometric shapes (cubes, pyramids and cylinders) using a multilayer neural network. The input data of the algorithm are the images of shapes placed in different positions and distances from the camera. The classification is based on feature vectors that are obtained using the methods of digital image processing. Feature vectors are inputs of neural network [1].

Hong-Nghi Tran and Tuan Nguyen have presented automatic number plate recognition software on Android smart-phone which utilizes the MLP neural network for number plate and character recognition [3].

Problems of different light conditions in image retrieval are solved in the contribution of the authors Affix Mareta, Indah Soesanti and Oyas Wahyunggoro. The article is focused on recognition of leaves which does not consider the light intensity and is not captured on the white background. There are different approaches to and accuracy levels of leaf segmentation in natural environment, and the level of complexity keeps increasing. The morphological features were used for the shape feature extraction. Local binary pattern was used for texture features extraction. Then the identification of accuracy was obtained by using Multilayer Perceptron algorithm (MLP). The contribution of this study is using proposed image enhancement and segmentation algorithm, so that the features of the image can be extracted based on the shape and texture descriptors [4].

The similar problems are defined in the article of authors Faner Meng, Heng Zheng and Zhezhou Yu. They deal with the recognition technology of the print code on beer bottles, using the method of processing and analysis of static image. Collecting the video information which contains the beer production date in the beer production line was done using the technology of image processing and image recognition. To ensure the quality of the products, the system in real-time recognizes the information of the print code on the beer bottles and eliminates the product whose print code is fuzzy or even wrong from the assembly line [5].

In other paper [6], a new method is proposed rotating the character image to the horizontal position and using the opening operation of morphology to cut the touching characters.

The problem of recognition is also solved by the QR code recognition. The paper of authors in [8] proposed a recognition method for low resolution QR-codes, applying a super-resolution 
technique. In the super-resolution process, the proposed method uses the property that QRcodes consist only of a binary pattern. Experimental results showed the effectiveness of the proposed method especially for recognizing low resolution QR- codes.

Similar problem of solving the QR code recognition caused by ordinary camera collection an the recognition algorithm based on image processing are investigated in contribution [9].

\section{PROBLEM DESCRIPTION AND SOLUTION}

As mentioned in the introduction, the core of this paper is the use of machine learning to recognize the position of a product when gripped by a robot in a manufacturing process. The entire production process can be described as follows: The robot carries out the assembly of products (double-acting piston) on a fully automated line. The piston itself consists of multiple elements. The main element is the body of piston. The piston body (Figure 1) is gripped by the robot during assembly, and then anchored into clamp. The entire piston is assembled step by step to its final state.

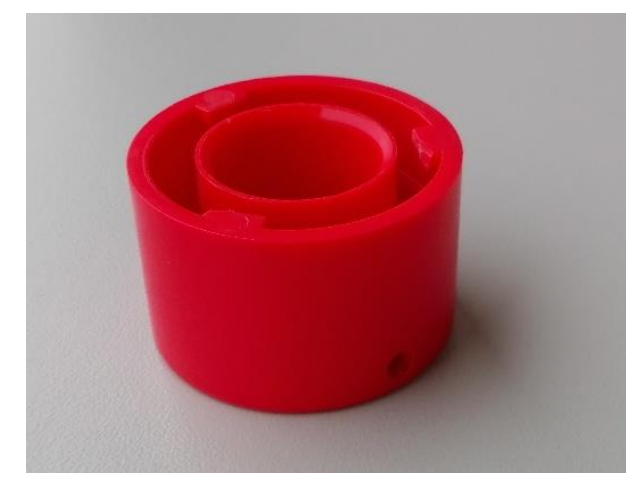

Figure 1 Piston body

The main problem is correct position of the piston body. The piston must be turned upwards. The detection of product position is performed via a CCD camera mounted to the robotic arm. The picture captured by camera is analysed using an MLP network. The output from neural network is simply "up" or "down", the reaction of robot to this information is turning the piston body by 180 degrees.

Generally, the biggest problem of the image recognition is daytime variability of lighting conditions. The proposed design is aimed at designing a system which would be highly reliable regardless of the direction, intensity and colour of the working environment. The picture in Figure 2 shows images of the same component displayed under different lighting conditions. The first group shows the example of pictures of piston body from the top (Figure 2), the second group shows it from the bottom (Figure 3). Moreover, it is obvious that the parts are differently axially rotated in each case.
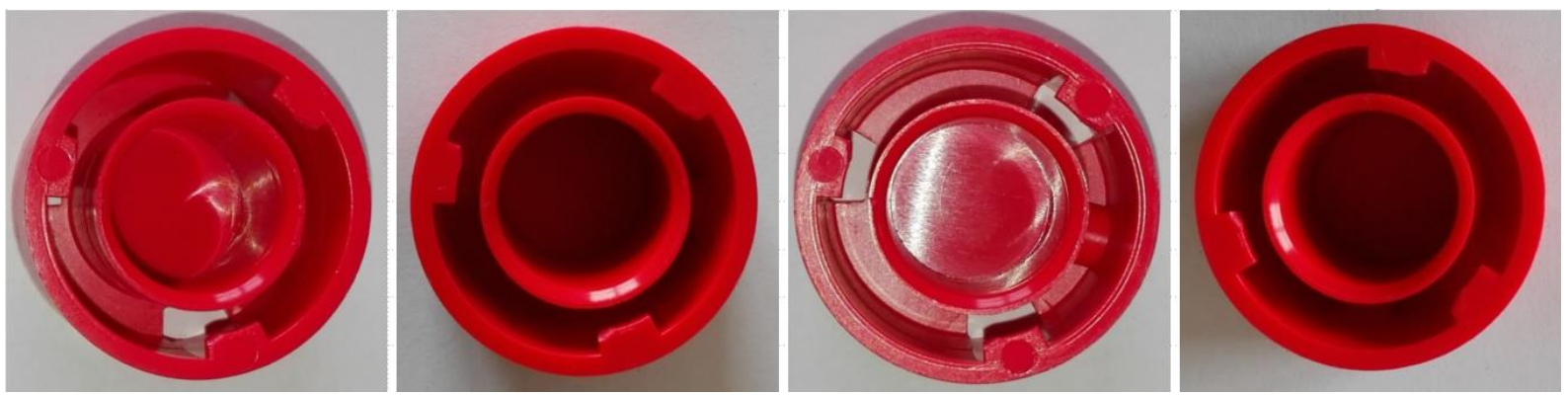

Figure 2 Example of recorded pictures of piston body from top - different lighting conditions 

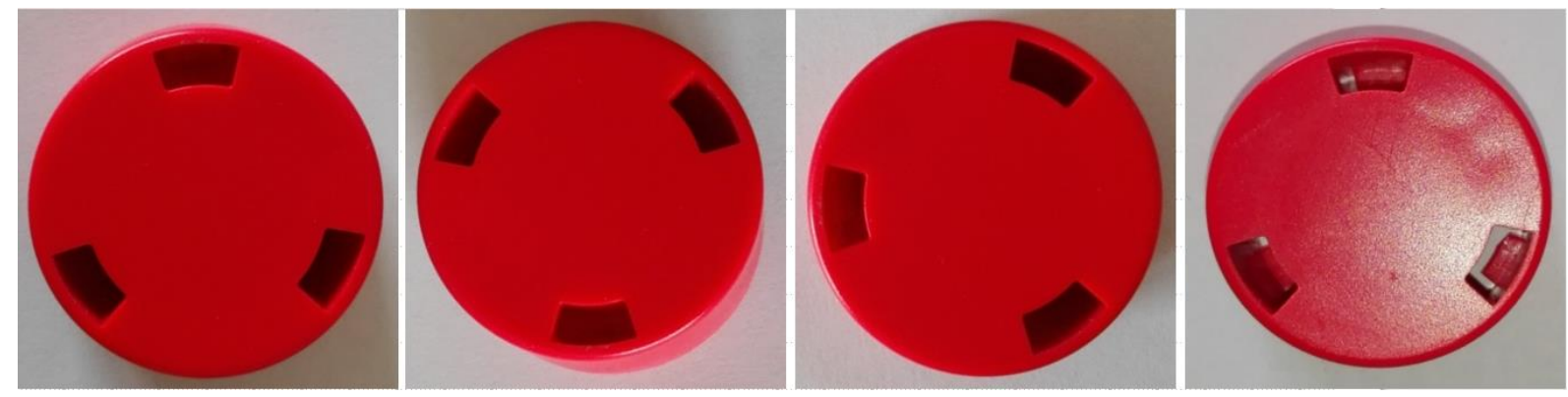

Figure 3 Example of recorded pictures of piston body from bottom - different lighting conditions

The whole system consists two parts. The first one is an optical part represented by a CCD camera positioned on the robotic arm, which captures colour image. The second part is a trained MLP neural network. The neural network in this experimental phase is designed in Matlab. The control computer with running Matlab communicates with control unit of the robot. Program in the robot PLC is designed to respond to output from control computer, and dynamically insert necessary subroutines for manipulation with components.

\section{Description of image data transformation}

Identification of the scanned image components is performed by the MLP neural network. The image data captured by the camera serves as input to the neural network. The structure of neural network strongly depends on the size and pixel resolution of captured pictures. In our case, quality of the scanned image was empirically chosen as a compromise between the sizes of data files, resolution and colour depth. Based on the experiments, the camera has been setted up to $800 \times 800$ pixels image, true colour -24 bit RGB. The image processing procedure is as follows: Firstly, the scanned image is transformed programmatically into a colour histogram. Next, information coded from pictures in histogram is used as input into neural network [10,11]. The process of developing the histogram is the result of the following procedure:

a) the representation of picture in each point with quantification of colour intensity

Each captured image is represented by the number of 800x800 pixels, i.e. the total of 640,000 pixels. The colour intensity of each pixel is defined by 8 bit information $\left(2^{8}\right)$ for every colour component $(\mathrm{R}, \mathrm{G}, \mathrm{B})$. That means:

$$
\begin{aligned}
& \operatorname{pixel}(1)[R, G, B]-[25,48,156] \\
& \operatorname{pixel}(2)[R, G, B]-[37,158,235] \\
& \ldots \ldots \\
& \operatorname{pixel}(640000)[R, G, B]-[154,25,68]
\end{aligned}
$$

b) Creation of attribute bits via binning the pixels

Every pixel in captured image is assigned to the bit group according to the brightness of the color component. If intensity of colour in each colour component (RGB) is lower than the limit value is represented as LOW. If value of specific colour is higher as threshold is represented as HIGH bit. Specified boundaries are shown in Table 1. 


\begin{tabular}{l}
\hline Table 1 Boundaries for pixel binning \\
\begin{tabular}{|c|c|c|c|c|c|c|}
\hline LOWR & HIGHR & LOWG & HIGHG & LOWB & HIGHB & Identification bin \\
\hline 0 & 127 & 0 & 127 & 0 & 127 & 1 \\
\hline 0 & 127 & 0 & 127 & 127 & 255 & 2 \\
\hline 0 & 127 & 127 & 255 & 0 & 127 & 3 \\
\hline 0 & 127 & 127 & 255 & 127 & 255 & 4 \\
\hline 127 & 255 & 0 & 127 & 0 & 127 & 5 \\
\hline 127 & 255 & 0 & 127 & 127 & 255 & 6 \\
\hline 127 & 255 & 127 & 255 & 0 & 127 & 7 \\
\hline 127 & 255 & 127 & 255 & 127 & 255 & 8 \\
\hline
\end{tabular}
\end{tabular}

This means, for example pixels following classification:

$\operatorname{pixel}(1)[R, G, B]-[25,48,156]-$ belongs to identification bin No. 2 . $\operatorname{pixel}(2)[R, G, B]-[37,158,235]$ - belongs to identification bin No. 4

$\operatorname{pixel}(640000)[R, G, B]-[154,25,68]-$ belongs to identification bin No. 5

The output of this transformation is matrix with values for every pixel in the picture. The graphical representation of the data is shown in histogram. Consequently, the data is joined to matrix and used as a set of input vectors to enter the neural network.
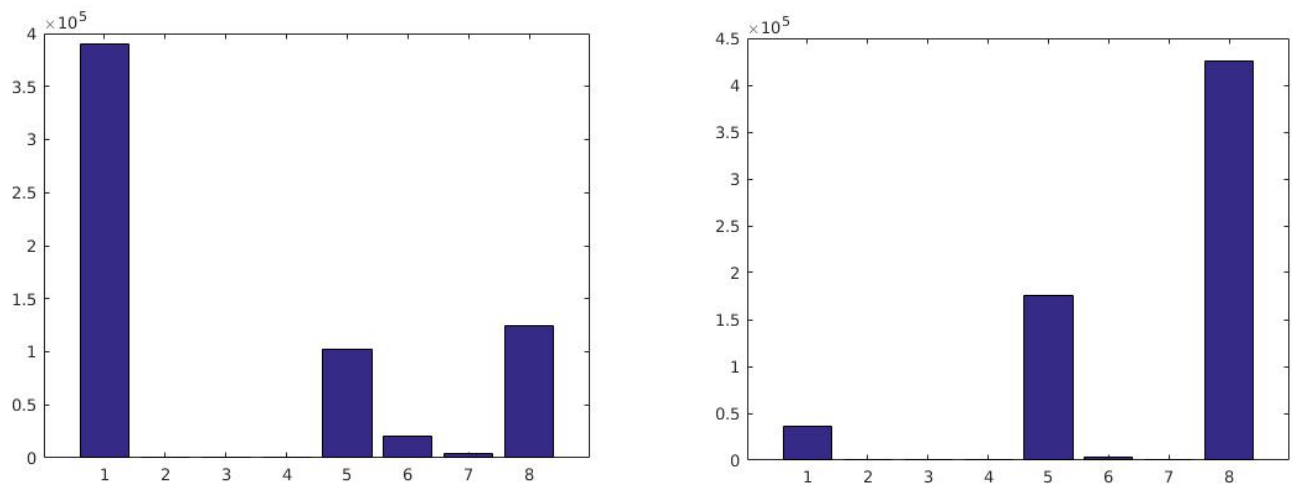

Figure 4 Examples of generated histograms

\section{Design of neural network}

Neural networks are, for their characteristics, widely used as classifiers for image recognition. There are many types of neural networks. In this case, Multilayer perceptron neural network was used. This type of neural network can be defined as a feedforward artificial neural network that generates a set of outputs from a set of inputs. An MLP is characterized by several layers of input nodes connected as a directed graph between the input and output layers. MLP uses backpropagation for training the network. MLP is a deep learning method. MLP is widely used for solving problems that require supervised learning, as well as research into 
computational neuroscience and parallel distributed processing. Applications include speech recognition, image recognition and machine translation [12].

For implementation simplicity in laboratory conditions, software Matlab was used along with the built-in modules for MLP. As input into neural network, matrix of inputs was used. The matrix consisted of calculated histograms of images shot by the camera on the robot arm. For MLP learning, matrix of desired outputs is also important.

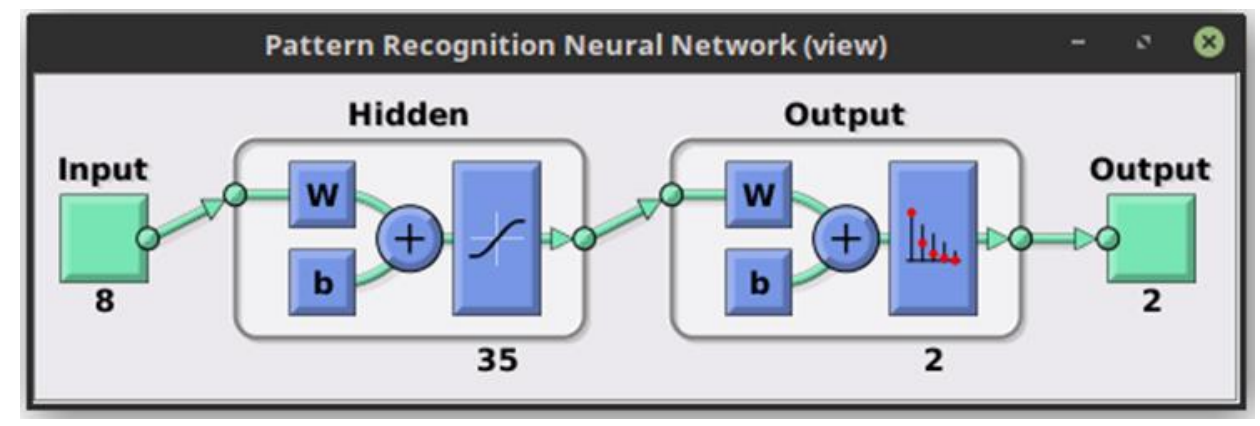

Figure 5 Neural network configuration

The entire neural network is implemented as a 3-layer neural network. Input layer is made up of eight neurons. These correspond to identification bins defined in transformation of image data. The network contains one hidden layer of 35 neurons. Although the network basically works as a simple two-class classifier and output is just one neuron/bit, in this case, the output layer is made up of two neurons. The identification of a component position is represented by combination of output neurons/bits. Output $[0,1]$ means that piston body is turned up, output $[1,0]$ means that piston body is turned down.

Number of pictures for training and testing the neural network was divided into two groups. Firstly, the training-set was set to 1000 pictures for learning the neural network. Secondly, a group of 100 pictures was chosen for testing.

After many tests, we considered that the best algorithm seems to be the scaled conjugate gradient algorithm (trainscg) [13].

This algorithm does not perform a line search at each iteration, and the training stops when any of these conditions occur:

- the maximum number of epochs (repetitions) is reached,

- the maximum amount of time is exceeded,

- performance is minimized to the goal,

- the performance gradient falls below min_grad,

- validation performance increased more than max_fail times since the last time it decreased (when using validation).

After several attempts with different types of algorithm and structures of neural networks, neural network was designed using this learning method. The presented neural network is capable of achieving an accuracy of $98.9 \%$. Outputs from confusion matrix are shown in Figure 6. 


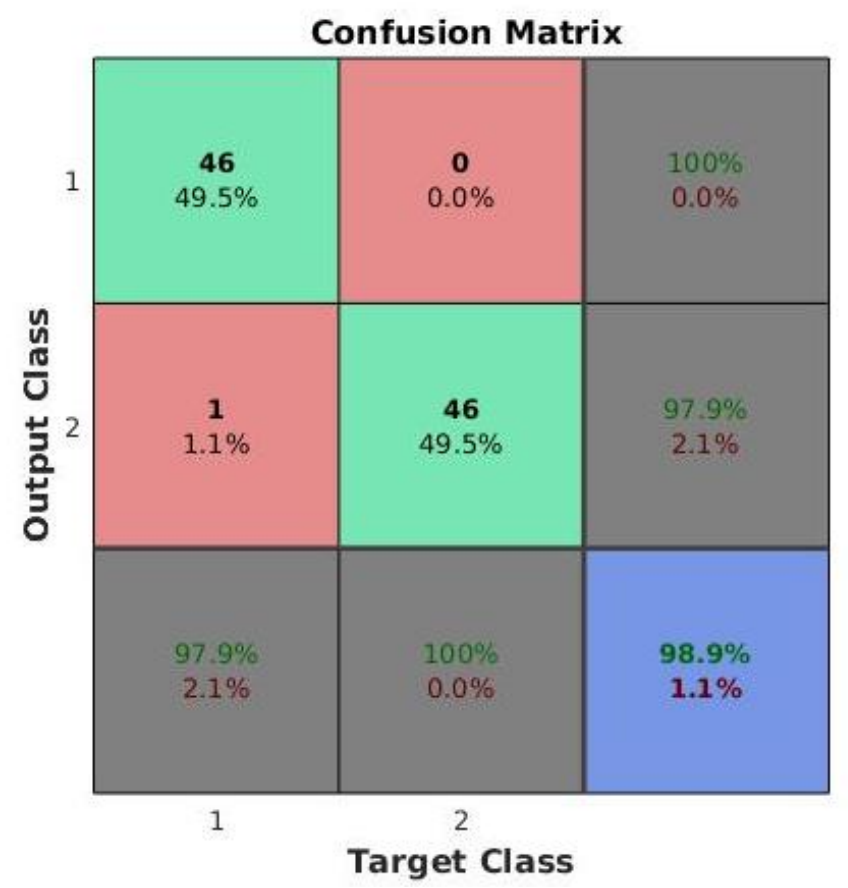

Figure 6 Confusion matrix from trained MLP

\section{IMPLEMENTATION OF THE SOLUTION IN THE TESTING ENVIRONMENT}

After verification of functionality of designed method in virtual environment in computer, practical experiments were performed. The entire system in fact consists of the Mitsubishi RV2FB-D robotic arm with the CR750-D control unit, together with a computer for real-time processing of the camera image.

The control computer is joined with the robot's base unit via ethernet interface using TCP/IP communication protocol. Generally, it is assumed that a switched ethernet network is not suitable for real-time process control. This premise in this case is not important, which is due to the fact, that control program is created in such a way, that robot is always waiting for decision from neural network.

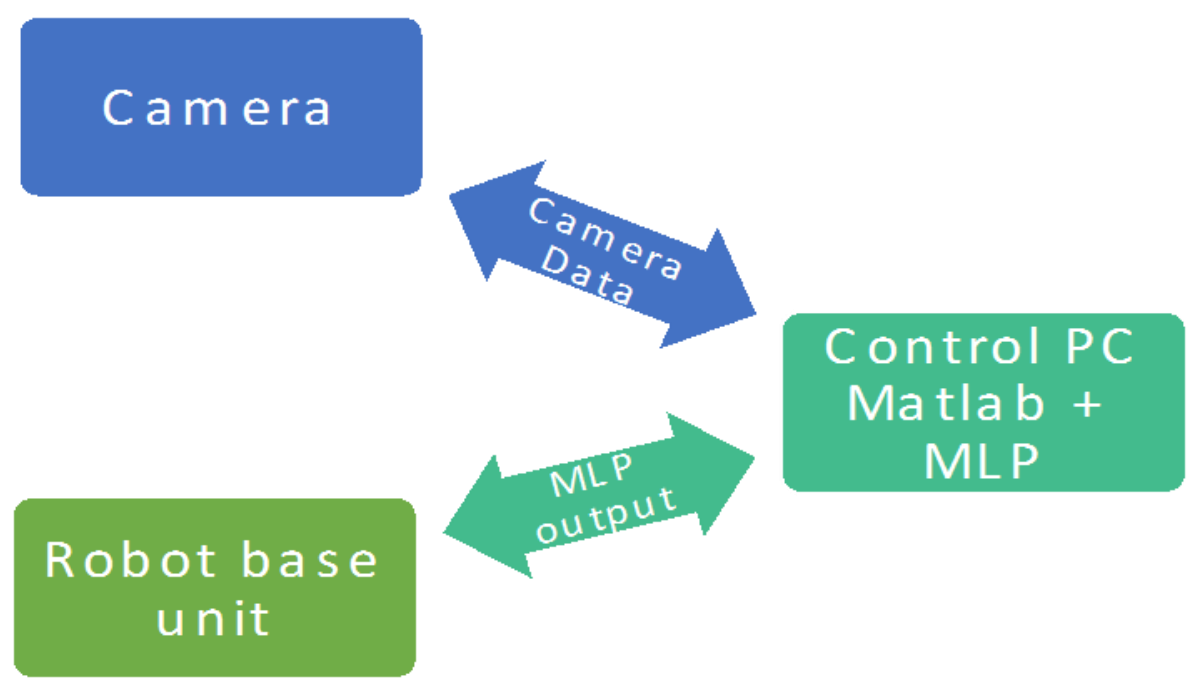

Figure 7 Data flow in designed system 
The practical implementation of designed system with the camera on the robot arm, along with the overall layout of the workplace is shown in Figure 8.

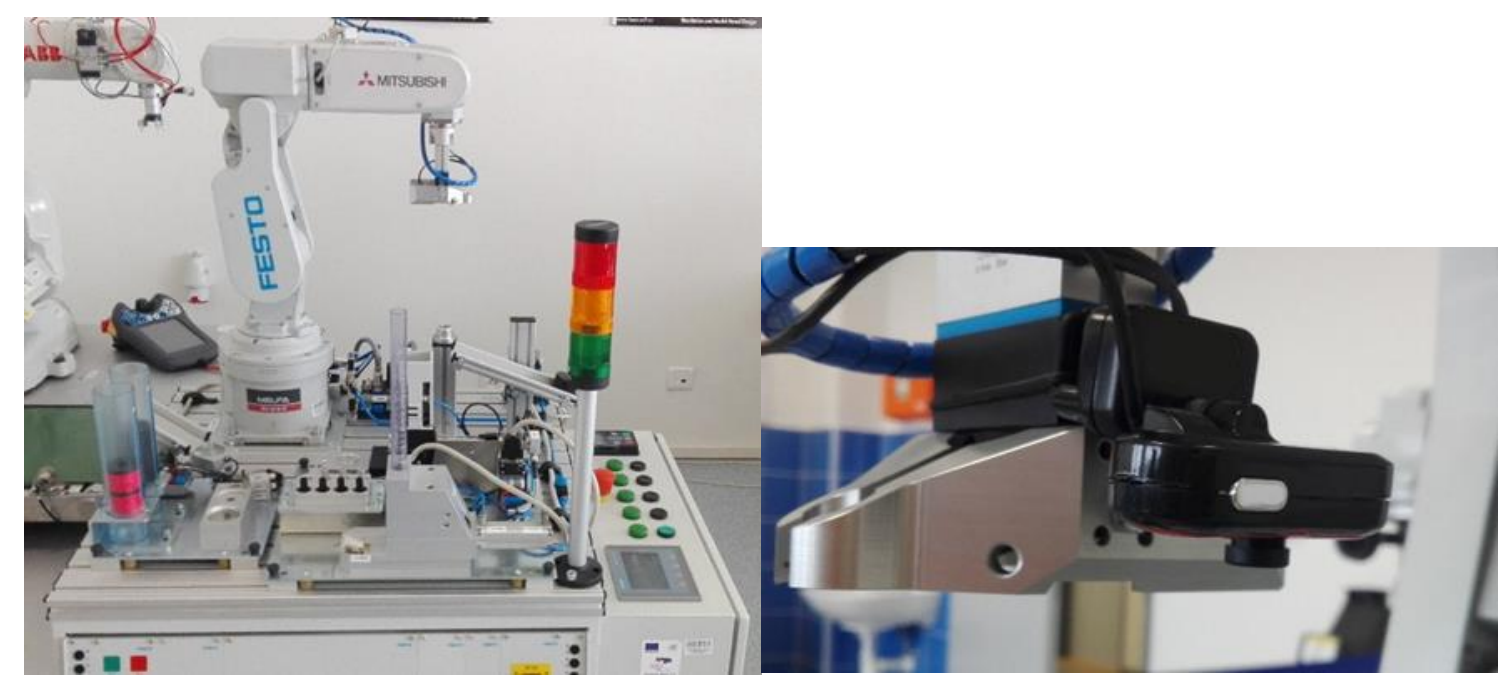

Figure 8 Example of practical realization

\section{CONCLUSION}

The core of this paper is the application of well-known technologies of neural networks to control a robotized assembly line. Though this type of technology is well known and often used in practice, the described solution has several positive aspects. Thanks to the used neural network, detection of components' positions is highly reliable. Remarkably, the recognition does not depend on the rotation of the piston body or the colour illumination. In addition, according to practical tests, recognition does not depend on the reflection and the angle of incidence of the light beams. This is particularly important in the light conditions in the areas with natural light (sunlight impact). In addition, tests were carried out with the parts of different colour. They showed that the algorithm is virtually insensitive to colour change due to the luminance component only. This means, the suggested system recognizes blue pistons with minimal adjustment - by changing the sensitivity coefficient in the program - and also, for example, black pistons.

In conclusion, the whole system is simple to implement, efficient in application and easy to implement in practice thanks to its recognition capabilities, combined with the use of a relatively simple neural network.

\section{Acknowledgement}

This publication was written with the financial support of the VEGA agency in the frame of the Project 1/0232/18 "Using the methods of multi-objective optimization in production processes control".

This publication has been written thanks to support of the Operational Program Research and Innovation for the project Research, modeling and simulation of industrial production processes using progressive technologies, ITMS code: NFP313010T589 co-financed by the European Regional Development Fund. 


\section{References}

[1] SPASOJEVIĆ, S. S., ŠUŠIĆ, M. Z., DUROVIĆ, Ž. M. 2012. Recognition and classification of geometric shapes using neural networks. In: 11th Symposium on Neural Network Applications in Electrical Engineering. NEUREL-2012, Faculty of Electrical Engineering, University of Belgrade, Serbia, pp. 71-76. ISBN 978-1-4673-1572-2.

[2] YANG, Y., ZHENG, P., HE, H., ZHENG, T., WANG, L., HE, S. 2018. An Evaluation Method of Acceptable and Failed Spot Welding Products Based on Image Classification with Transfer Learning Technique. In: ACM International Conference Proceeding Series. 2nd International Conference on Computer Science and Application Engineering: CSAE 2018, Hohhot China, a109. ISBN 978-145036512-3.

[3] TRAN, H. N., NGUYEN, T. 2014. Supervised learning method for mobile international number plate recognition. In: ACM International Conference Proceeding Series. 16th International Conference on Information Integration and Web-Based Applications and Services: iiWAS 2014, Hanoi, Viet Nam, pp. 212-216. ISBN 978-145033001-5.

[4] MARETA, A., SOESANTI, I., WAHYUNGGORO, O. 2018. Herbal Leaf Classification Using Images in Natural Background. In: 2018 International Conference on Information and Communications Technology. 1st International Conference on Information and Communications Technology: ICOIACT 2018, Grand Zuri, Yogyakarta, Indonesia, pp. 612-616. ISBN 978153860954-5.

[5] MENG, F., ZHENG, H., YU, Z. 2011. Extracting and Recognition of Print Code on Beer Bottle Based on Regional Analysis. In: 2011 International Conference on Electronics, Communications and Control. ICECC 2011, Ningbo, China, pp. 3746-3749. ISBN 978-145770321-8.

[6] YU, F.-C., LU, L.-K. 2010. The Application of Computer Vision Algorithms in Touching Characters Recognition of Industry Products. In: 2010 International Conference on Mechanic Automation and Control Engineering. MACE 2010, Wuhan, China, pp. 523-527. ISBN 978142447738-8.

[7] LEE, S. J., KIM, S. W. 2016. Recognition of Slab Identification Numbers Using a Deep Convolutional Neural Network. In: 15th IEEE International Conference on Machine Learning and Applications. IEEE ICMLA 2016, Anaheim, California, USA, pp. 718-721. ISBN 978-15090-6167-9.

[8] KATO, J., DEGUCHI, D., TAKAHASHI, T., IDE, I., MURASE, H. 2011. Low resolution QRcode recognition by applying super-resolution using the property of QR-codes. In: 2011 International Conference on Document Analysis and Recognition. ICDAR 2011, Beijing, China, pp. 992-996. ISBN 978-1-4577-1350-7

[9] GU, Y., ZHANG, W. 2011. QR Code Recognition Based On Image Processing. In: 2011 International Conference on Information Science and Technology. ICIST 2011, Nanjing, China, pp. 733-736. ISBN 978-1-4244-9442-2.

[10] https://mpatacchiola.github.io, Massimiliano Patacchiol: "The Simplest Classifier: Histogram Comparison“. [Online]. [Accessed: 07. - 2019] Available at: https://mpatacchiola.github.io/blog/2016/11/12/the-simplest-classifier-histogramintersection.html

[11] WHEELER, A, HENRIQUES, R. 2017. Standard and Super-Resolution Bioimaging Data Analysis: A Primer. John Wiley \& Sons, 2017 - 312 pages, ISBN:1119096936

[12] www.techopedia.com, "Multilayer Perceptron (MLP)“. [Online]. [Accessed: 07.-2019] Available at: https://www.techopedia.com/definition/20879/multilayer-perceptron-mlp

[13] MEILLER, M.F. 1993. A Scaled Conjugate Gradient Algorithm for Fast Supervised Learning. Neural Networks, Vol. 6, Issue 4, pp. 525-533. ISSN: 0893-6080

\section{ORCID}

Igor Halenár

Gabriela Križanová
$0000-0002-5445-3625$

0000-0002-5699-6732 